Int. J. Dev. Biol. 58: 349-353 (2014)

doi: $10.1387 / \mathrm{ijdb} .140016 \mathrm{yl}$

\title{
Dipeptidyl peptidase IV reduces trophoblast invasion by inhibiting the activity of MMPs
}

\author{
YOUFEI LI*,1 ${ }^{*}$ ZHEN LI ${ }^{1}$ and JIAN ZHANG ${ }^{2}$ \\ ${ }^{1}$ Department of Obstetrics and Gynecology, Xinqiao Hospital and ${ }^{2}$ Department of Pathobiology, Third Military Medical \\ University, Chongqing, China
}

\begin{abstract}
Preeclampsia is a severe pregnancy complication in part due to insufficient implantation. This study aimed at elucidating the mechanism of action of dipeptidyl peptidase IV (DPPIV) in preeclampsia. Small activating RNAs (saRNA) were used to upregulate DPPIV expression in human trophoblast JAR cells. The DPPIV expression level was analyzed by real-time quantitative PCR and western blot and its activity was measured by luminescent protease assay. MMP-9 activity was analyzed by zymography and cell invasion by matrigel invasion assay. DPPIV expression level and activity was significantly increased by saRNA in JAR cells. DDPIV specific inhibitor diprotin $A$ inhibited its activity at both basal and activated levels. DPPIV did not regulate MMP-9 expression but did repress MMP-9 activity. The invading ability of JAR cells was reduced by saRNA but increased by diprotin A. DPPIV might be responsible for the shallow implantation of the placenta due to its inhibition of the invading ability of extravillous trophoblasts, causing preeclampsia at later stage of pregnancy.
\end{abstract}

KEY WORDS: preeclampsia, trophoblast, dipeptidyl peptidase IV, small activating RNA, cell invasion, diprotin A

Preeclampsia is a severe complication which affects $5-8 \%$ of pregnancies and contributes significantly to maternal and fetal morbidity and mortality (Steegers et al., 2010). Moreover, women with preeclampsia have an increased risk of developing cardiovascular disease later in life. Preeclampsia is thought to be resulted from a complex interplay between genetic and environmental factors. Family studies provides the evidence for the involvement of genetic factors, which have shown that daughters and sisters of preeclamptic women have a higher risk of preeclampsia (Nilsson et al., 2004). Moreover, the incidence of preeclampsia is also different among various ethnic groups (Steegers et al., 2010). Unfortunately, the precise etiology of preeclampsia remains unclear after decades of efforts.

It is required for a blastocyst to implant into the uterine endometrium to form a functional placenta during the establishment of mammalian pregnancy. A functional placenta formation requires the 'extravillous trophoblast' (EVT) engrafting and remodeling uterine spiral arteries to create placental blood supply at the end of the first trimester (Burton et al., 2010). Shallow or inappropriate implantation and placentation may result in first trimester miscarriage, preeclampsia, and other obstetric complications (Aplin, 2010). The trophoblast invasion is regulated by local endometrial environment that endometrial stromal cells differentiate into decidual cells, becoming rounded with altered expression and secretion of extracellular matrix (ECM) (Menkhorst et al., 2012).

The degradation of extracellular matrix (ECM) plays a critical role in cell migration, invading, and engraftment. Matrix metalloproteinases (MMPs) are zinc-dependent endopeptidases capable of degrading components of the ECM. MMP-9 has been shown to play roles in tissue breakdown and remodeling during aggressive tumor growth and angiogenesis (Halade et al., 2013). The upregulation of MMP-9 associates with the increased motility of bovine trophoblast cells (Dilly et al., 2010).

Dipeptidyl peptidase IV (DPPIV), also known as CD26, is differentially expressed in different cell population in human placenta and fetal membrane alone pregnancy. In the first trimester, DPPIV expression was mostly found in the proximal part of the cell column and undetectable in interstitial trophoblasts (Sato et al., 2002). Diprotin A (Ile-Pro-Ile) is a specific competitive inhibitor of DDPIV that binds to the substrate-binding site of PPIV (Hiramatsu et al., 2004) and significantly enhances engraftment of human mobilized CD34+ peripheral blood cells in mice (Kawai et al., 2007). Placental

Abbreviations used in this paper: DPPIV, dipeptidyl peptidase IV; ECM, extracellular matrix; EVT, extravillous trophoblast; MMP, matrix metalloproteinase; saRNA, small activating RNA.

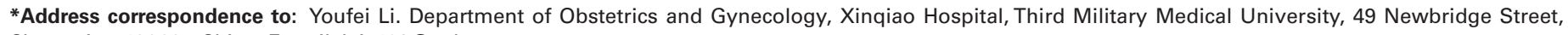
Chongqing 400037, China. E-mail: leis428@yahoo.com
}

Accepted: 13 June, 2014. Final, author-corrected PDF published online: 30 September 2014.

ISSN: Online 1696-3547, Print 0214-6282 
A

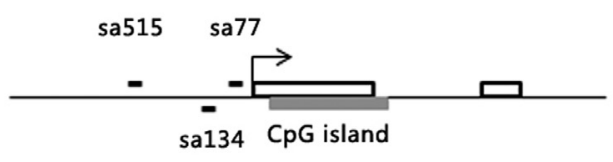

B

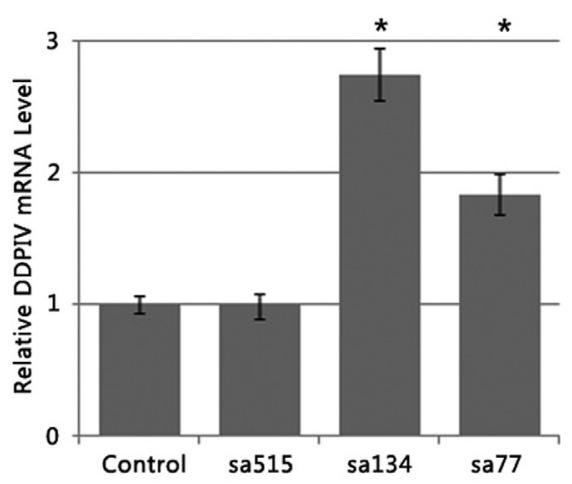

C

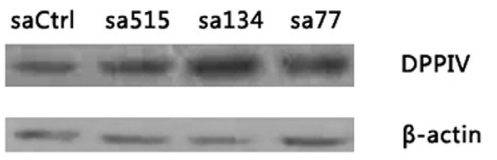

Fig. 1. Human DPPIV expression was activated by saRNA. (A) A diagram shows the three saRNA targets in relation with the transcription start site. (B) DPPIV mRNA levels in JAR cells treated with specific saRNA, the level of control cells was set as 1. (C) The protein levels of DPPIV from JAR cell treated with different saRNA. ${ }^{*}: p<0.01$ compared with control.

DPPIV activity was found to be significantly higher in preeclamptic placentas with intrauterine growth restriction compared to control (Nishikawa et al., 2005), indicating that DPPIV activity might be a risk factor for preeclampsia. Increased decidual DPPIV positive lymphocytes was reported to be associated with miscarriage in human (Sehmsdorf et al., 2004). This study aimed at elucidating the roles and relationship of DDPIV and MMPs in EVT invasion.

\section{Results}

\section{Small RNA activates human DPPIV expression}

The nucleotide sequences for the hairpin structure of three small RNAs were designed to target different regions of human DPPIV promoter (Fig. 1A). The nucleotides were cloned into lentiviral vector PLL3.7 under the control of U6 promoter and viruses were generated in 293T cells. Two of the small RNAs activated DPPIV expression at both mRNA (Fig. 1B) and protein (Fig. 1C) levels. The small activating RNA targeting human DPPIV promoter region starting from -134 base pair produced highest expression levels of DPPIV (Fig. 1A and 1B), which was chosen for later experiments and named saDPPIV.

\section{Diprotin A inhibits saRNA induced DPPIV activity, but has no effect on DPPIV expression}

To determine the effect of DPPIV inhibitor diprotin A on the expression level and activity of DPPIV in saRNA treated JAR cells, the mRNA, protein, and protease activity of DPPIV in the presence or absence of diprotin A were analyzed. In JAR cells, diprotin A did not significantly change either mRNA (Fig. 2A) or protein (Fig. 2B) levels of DPPIV independent of the presence of small activating RNA. However, diprotin A markedly reduced DPPIV enzymatic activity (Fig. 2C). The DPPIV activity was significantly increased in saDPPIV cells which was repressed by diprotin A treatment (Fig. 2C).

\section{DPPIV inhibits MMP-2 and MMP-9 activities}

The MMP-9 mRNA and protein levels in JAR cells were not significantly changed by DPPIV promoter activating small RNA compared to that of control cells or by diprotin A treatment (Fig. 3 A ,B). The extracellular MMP-2/9 activities analyzed by zymography showed significant change by both saDPPIV and diprotin (Fig. 3 C,D). The degradation of gelatin by MMP-2/9 was markedly stronger by diprotin A treated JAR cells but saDPPIV inhibited gelatin degradation.

\section{DPPIV inhibits invasion of JAR cells}

Neither lentiviruses nor working diprotin A had significant effect on the proliferation rate of JAR cells (Fig. 4A). Diprotin Asignificantly increased the number of invading JAR cells in a dose dependent fashion whereas saDPPIV markedly reduced the number of invading cells compared to control (Fig. 4 B,C). The treatment of diprotin A effectively relieved the inhibition of saDPPIV on the invasion of JAR cells ( $p<0.01$ saDPPIV vs saDPPIV/diprotin) (Fig. $4 C$ ).

\section{Discussion}

In human choriocarcinoma cell line JAR, DPPIV expression was significantly increased by small activating RNA targeting its proximal promoter regions, with significant increase of DPPIV mRNA, protein, and activity levels. DPPIV inhibitor diprotin A inhibited DPPIV enzymatic activity but had no impact on the expression level of DPPIV. MMP-2/9 activities and the invading ability of JAR cells were inversely correlated with DPPIV level in JAR cells.
A

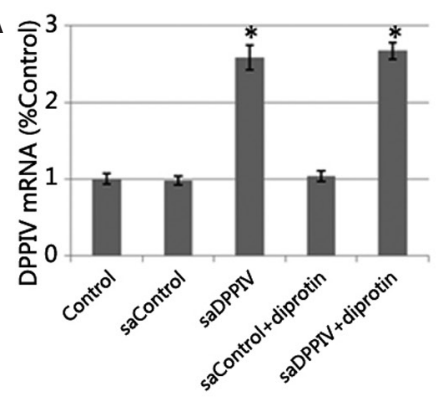

B

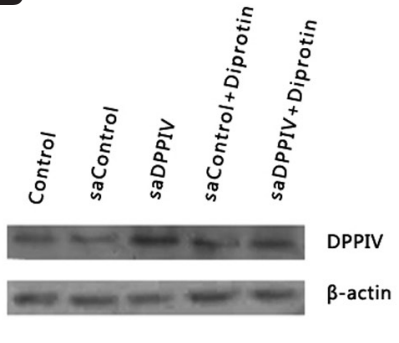

C

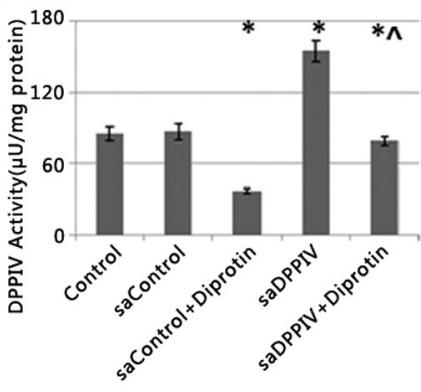

Fig. 2. Diprotin A inhibits saRNA induced DPPIV activity but has no effect on DPPIV expression. The mRNA (A), protein (B), and dipeptidase activity (C) levels of JAR cells treated with saRNA and Ior diprotin $A .{ }^{*}: p<0.01$ compared with control; $\wedge: p<0.01$ compared with saDPPIV. 
A



B

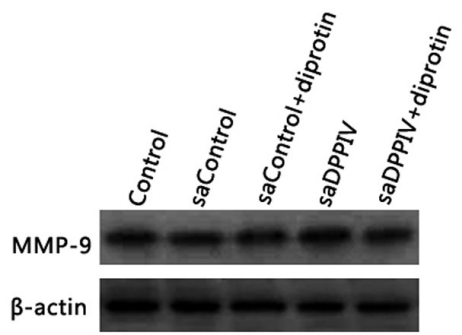

C

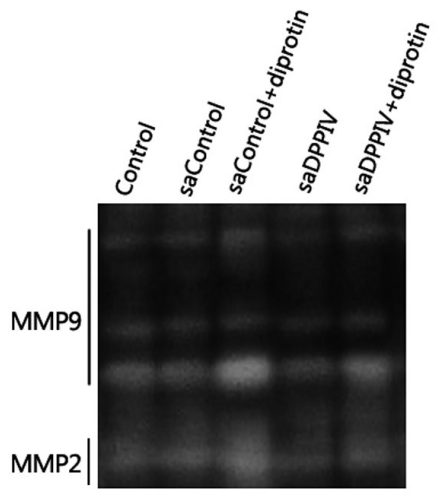

D

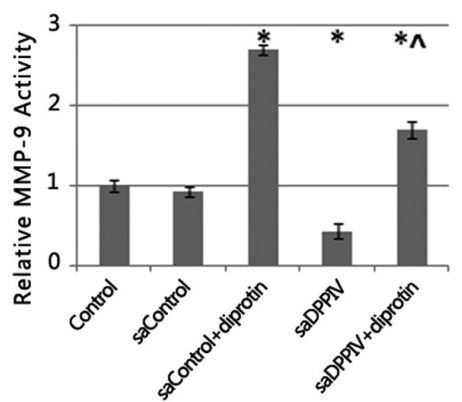

Fig. 3. DPPIV inhibitsMMP-9 activity. (A) MMP-9 mRNA levels in JAR cells treated with saRNA and / or diprotin A analyzed by RT-qPCR. (B) The activity of MMP-9 of saRNA and I or diprotin A treated JAR cells was assayed by zymography. (C,D) Quantitative analysis of MMP-9 activity. ${ }^{*}: p<0.01$ compared with control; $\wedge$ : $p<0.01$ compared with saDPPIV.

A

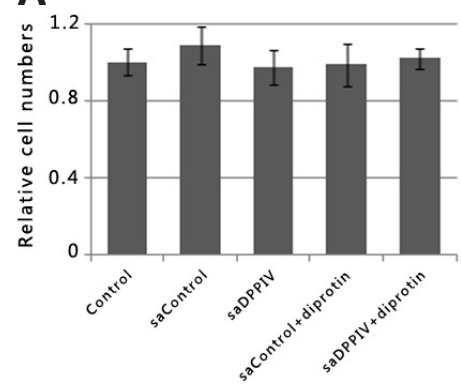

B

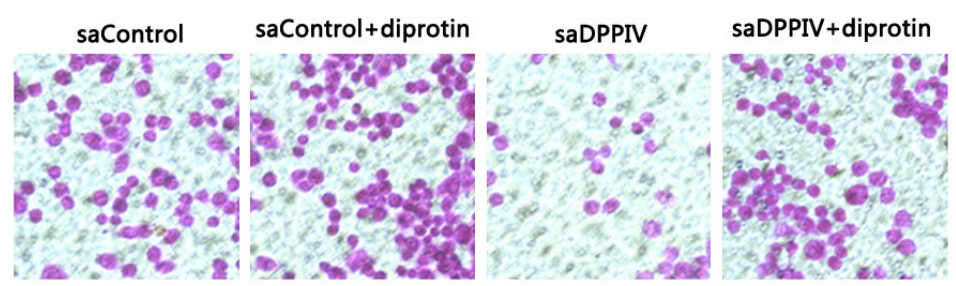

Fig. 4. DPPIV inhibits invasion but not growth of JAR cells. JAR cells were infected with saControl or saDPPIV lentivirus, then treated with or without $5 \mathrm{ng} / \mathrm{ml}$ or $20 \mathrm{ng} / \mathrm{ml}$ diprotin A. (A) MTT results showed that neither diprotin A nor lenti-saDPPIV significantly changed the growth of JAR cells. (B) Representative pictures of invading JAR cells with only $20 \mathrm{ng} /$ $\mathrm{ml}$ diprotin A treatment showing here. (C) Average invading JAR cells per field from saRNA and / or diprotin A treatment. * : $p<0.01$ compared to control; \#: $p<0.01$ compared with 5 $\mathrm{ng} / \mathrm{ml}$ diprotin $A ; \$: p<0.01$ compared with saDPPIV; 1 : $p<0.01$ compared with saDPPIV/5 $n g / m /$ diprotin $A$.
RNA activation strategy has been shown to efficiently induce some tumor suppressor genes in various cancers in vitro and in vivo (Kang et al., 2012), which inhibited cancer cell proliferation and tumor growth. The saRNA-induced E-cadherin overexpression resulted in the suppression of migration and invasion of human bladder cancer cells in vitro (Kang et al., 2012). DPPIV expression level was elevated in JAR cells by lentivirus-delivered saRNA targeting DPPIV gene promoter regions.

Systemic DPPIV inhibition with sitagliptin enhanced engraftment during umbilical cord blood (UCB) transplantation in adult with hematological malignancies (Farag et al., 2013), which might be due to the reverse of DPPIV-caused downregulation of CXCL12-induced chemotactic response (Christopherson et al., 2006). On the other hand, DPPIV was found to mediate granulocyte colonystimulating factor mobilization of hematopoietic stem and progenitor cells (Broxmeyer et al., 2007). Moreover, DPPIV and other adhesion molecules on syncytiotrophoblast microvillous membranes were able to inhibit the proliferation of cultured human umbilical vein endothelial cells (HUVEC) (Kertesz et al., 2000) which was suspected through competition for ligands in ECM or serum with receptors on HUVEC. It seemed that DPPIV might have different roles other than chemotaxis in placenta. Current data demonstrated that DPPIV might inhibit trophoblast invasion during placenta implantation by inhibiting the activities of specific MMPs. The N-terminus of human MMP-9 proprotein starts with APRQRQST and MMP-2 with APSPIIKF. Xaa-Pro is the preferred substrate of DDPIV (Scharpé et al., 2001), indicating that the activities of MMP-2 and MMP-9 might be regulated through a process initiated by $\mathrm{N}$-terminus truncation by DDPIV.

It has been shown that DPPIV suppresses the growth and progression of many cancer cells (Wesley et al., 2004) but also found overexpressed in type II diabetes, chronic liver diseases, and various malignant tumors (Itou et al., 2013), indicating its function varies among different tissues and cells. DPPIV mediates G-CSF mobilization, engraftment, and survival of hematopoietic stem and progenitor cells (Broxmeyer et al., 2007) but suppresses NSCLC cells (Wesley et al., 2004). By using saRNA and DPPIV inhibitor diprotin $A$, an inverse relationship between DPPIV activity and the invading ability of human choriocarcinoma JAR cells was established. This line of data is consistent with in vivo findings that DPPIV was highly expressed in the EVTs of the proximal part of the cell column or into deep section of the decidua and myometrium but turned off in the migrating EVTs (Sato et al., 2002). Furthermore, DPPIV might inhibit extravillous trophoblast invasion through repressing the activity of certain ECM degrading enzyme such as MMP-2 and MMP-9. 
In conclusion, human DPPIV expression was activated by small activating RNA that targets specific region in DPPIV promoter. Increased expression level and activity of DPPIV repressed the activities of MMP-2 and MMP-9 and decreased JAR cell invading ability, indicating that dysregulation of DPPIV expression might be responsible for abnormal implantation of placenta and causes preeclampsia at later stage of pregnancy.

\section{Materials and Methods}

\section{Cell culture}

Human choriocarcinoma cell line JAR was obtained from the American Type Culture Collection (Manassas, VA). The cells were maintained in Dulbecco's modified Eagle's medium/Ham's nutrient mixture F12 (Invitrogen, Shanghai, China) supplemented with 10\% FBS (Hyclone, Beijing, China), $100 \mathrm{IU} / \mathrm{ml}$ penicillin, and $100 \mu \mathrm{g} / \mathrm{ml}$ streptomycin (Invitrogen, Shanghai, Chian) at $37^{\circ} \mathrm{C}$ in a humidified atmosphere $\left(5 \% \mathrm{CO}_{2}\right)$. Diprotin A was added at a specified final concentration where indicated.

\section{Design and verification of saRNA}

Three small activating RNA candidates were designed targeting different regions of human DDPIV gene promoter. The nucleotide sequences of the synthesized polynucleotides were: Sa77: TGCCATTCAAGAGGGAACAATCTTCAAGAGAGATTGTTCCCTCTTGAATGGCTTTTTTC and tcgaGAAAAAAGCCATTCAAGAGGGAACAATCTCTCTTGAAGATTGTTCCCTCTTGAATGGCA; sa134: TGCAAGACAATCGGGAAATTCCTTCAAGAGAGGAATTTCCCGATTGTCTTGCTTTTTTC and tcgaGAAAAAAGCAAGACAATCGGGAAATTCCTCTCTTGAAGGAATTTCCCGATTGTCTTGCA; sa515: TGCACATTGTAACCACTCAAACTTCAAGAGAGTTTGAGTGGTTACAATGTGCTTTTTTC and tcgaGAAAAAAGCACATTGTAACCACTCAAACTCTCTTGAAGTTTGAGTGGTTACAATGTGCA. Each pair were annealed and cloned into $\mathrm{Xhol} / \mathrm{Hpal}$ digested pLL3.7 lentiviral vector and confirmed by DNA sequencing (Invitrogen, Shanghai, China). Three saRNA and saControl viruses were packaged in 293T cells in the presence of three packaging vectors. The lentiviral vector cloning and lentivirus production were completed by Chi Biotechnology (Jiangyin, China). The viral titer was assayed by serial dilution and infection of 293T cells followed by fluorescence-activated cell sorting (FACS) analysis. Four viruses individually infected JAR cells and RNA was analyzed by RT-qPCR 3 days later.

\section{Quantitative real-time $P C R$}

JAR cells infected with control or saDPPIV lentivirus at 5 multiplicity of infection when they reached about $90 \%$ confluence. Twenty-four hr postinfection, the cells were treated with $10 \mu \mathrm{mole} / \mathrm{L}$ of diprotin A or vehicle for $24 \mathrm{hr}$ before collected for RNA or protein work. Total RNA was extracted from cells using Trizol reagent (Invitrogen, Shanghai, China) according to manufacturer's instruction. RNA concentration and purity were analyzed by UV spectrophotometer. $1 \mu \mathrm{g}$ of total RNA was reverse transcribed into cDNA according to kit instructions (Transgen, Beijing, China). After 1:3 dilution of $c D N A$ with $\mathrm{ddH}_{2} \mathrm{O}, 2 \mu \mathrm{l}$ of diluted cDNA was used as template for real-time PCR using 2xTransStart Green qPCR supermix (Transgen, China). Primers used were designed with Primer3 online software. AGTGGCGTGTTCAAGTGTGG and CAAGGTTGTCTTCTGGAGTTGG for DPPIV; ACGACGTCTTCCAGTACCGA and CTGGTTCAACTCACTCCGGG for MMP9; TCCATGACAACTTTGGTATCG and TGTAGCCAAATTCGTTGTCA for GAPDH (internal control). Fluorescence quantitative PCR was performed on an ABI 7300 (Life Technologies, Carlsbad, CA). The relative expression level was calculated by $2^{<<t}$ method.

\section{Western blot}

The protein was resolved by SDS-polyacrylamide gel electrophoresis before transferred onto a PVDF membrane. The membrane was blocked with $5 \%$ non-fat milk and immunoblotted with anti-DPPIV antibody (ab28340,
Abcam, Cambridge, MA), followed by secondary antibody incubation. An enhanced chemiluminescence (ECL) (Sangon, Shanghai, China) method was used to detect the protein bands.

\section{DPPIV activity assay}

DDPIV protease activity in cells treated with saDPPIV in the presence or absence of diprotin A was measured by DPPIV-Glo ${ }^{\text {TM }}$ Protease Assay kit from Promega (Madison, Wisconsin) according to manufacturer's manual.

\section{MTT}

JAR cells were digested with $0.25 \%$ trypsin into single cell suspension and seeded into 96 -well plates at 2000 cells per well. The next day, the cells were infected with $5 \mathrm{MOI}$ of control or saDPPIV lentivirus followed by $24 \mathrm{hr}$ later being treated with $10 \mu \mathrm{mole} / \mathrm{L}$ of diprotin A for another $24 \mathrm{~h}$. Then $20 \mu \mathrm{L}$ of $0.5 \%$ 3-(4,5-dimethylthiazol-2-yl)-2,5-diphenyltetrazolium bromide (MTT) was added to each well and incubated for $4 \mathrm{~h}$ at $37^{\circ} \mathrm{C}$. The medium was carefully removed and $150 \mu \mathrm{L}$ of dimethyl sulfoxide (DMSO) was added to each well. The absorbance was measured at $492 \mathrm{~nm}$ on a microplate reader after 30 min incubation in dark at room temperature with shaking. The OD value of untreated cells was set as 1 . The experiment was performed 3 times independently in 6 replicates.

\section{Cell invasion assay}

Two days after saDPPIV or saControl lentivirus infections, $5 \times 10^{4}$ cells in serum-free media in the presence or absence of 5 or $20 \mu \mathrm{mole} / \mathrm{L}$ diprotin A were seeded into the upper chambers of a 24-well Matrigel invasion chamber (BD, Shanghai, China). Medium with $10 \%$ FBS was added to the lower chambers. Invading cells were fixed and stained with $\mathrm{H} \& \mathrm{E} 12 \mathrm{hr}$ later. The cell number from five high power fields of each treatment was counted.

\section{Gelatin zymography measure MMP-2/9 activities}

Protein was resolved on a $7.5 \%$ acrylamide gel co-polymerized with gelatin (BDH Chemical Co, Poole, UK) at a final concentration of $1 \mathrm{mg} /$ $\mathrm{ml}$ with a $4.5 \%$ acrylamide stacking gel. Gels were run in standard Trisglycine-SDS running buffer A SeeBlue molecular weight ladder (Invitrogen, Shanghai, China) was used for size determination. Gels were washed in water and wash buffer $\left(50 \mathrm{mmol} / \mathrm{L}\right.$ Tris- $\mathrm{HCl} \mathrm{pH} 8.0,5 \mathrm{mmol} / \mathrm{L} \mathrm{CaCl}_{2}$ and $2.5 \%(\mathrm{v} / \mathrm{v})$ Triton X-100) twice each with agitation at room temperature (30 minutes for each wash). The gels were then rinsed three times in water before incubating in $50 \mathrm{ml}$ of $1 \%$ (vol/vol) Triton X-100 in $20 \mathrm{mmol} / \mathrm{L}$ Tris- $\mathrm{HCl}, \mathrm{pH} 7.8$ and $5 \mathrm{mmol} / \mathrm{L} \mathrm{CaCl}_{2}$ at $37^{\circ} \mathrm{C}$ for 20 hours. The gel was scanned and analyzed with ImageJ software.

\section{Statistical analysis}

All experiments were performed three times. Data were expressed as mean \pm standard error or representative images were shown. The difference between treatment was analyzed by one-way ANOVAusing Graphpad Prism 6. It was considered statistically significant if $p$ value was less than 0.05 .

\section{Acknowledgements}

We would like to thank Dr. Shi Lei from Chi Biotechnology for critical reviewing of the manuscript. This work was supported by Natural Science Foundation of Chongqing (2010BB5189) and Natural Science Foundation Project of CQ CSTC.

\section{References}

APLIN JD (2010) Developmental cell biology of human villous trophoblast: current research problems. Int J Dev Biol 54: 323-329.

BROXMEYER HE, HANGOC G, COOPER S, CAMPBELL T, ITO S, MANTEL C (2007). AMD3100 and CD26 modulate mobilization, engraftment, and survival of hematopoietic stem and progenitor cells mediated by the SDF-1/CXCL12-CXCR4 axis. Ann N Y Acad Sci 1106: 1-19.

BURTON GJ, JAUNIAUX E, CHARNOCK-JONES DS (2010). The influence of the intrauterine environment on human placental development. Int J Dev Biol 54 303-312. 
CHRISTOPHERSON KW 2ND, URALIL SE, PORECHA NK, ZABRISKIE RC, KIDD SM, RAMIN SM (2006). G-CSF- and GM-CSF-induced upregulation of CD26 peptidase downregulates the functional chemotactic response of CD34+CD38human cord blood hematopoietic cells. Exp Hematol 34: 1060-1068.

DILLY M, HAMBRUCH N, HAEGER JD, PFARRER C (2010). Epidermal growth factor (EGF) induces motility and upregulates MMP-9 and TIMP-1 in bovine trophoblast cells. Mol Reprod Dev. 77: 622-629.

FARAG SS, SRIVASTAVA S, MESSINA-GRAHAM S, SCHWARTZ J, ROBERTSON MJ, ABONOUR R, CORNETTA K, WOOD L, SECREST A, STROTHER RM, JONES DR and BROXMEYER HE (2013). In vivo DPP-4 inhibition to enhance engraftment of single-unit cord blood transplants in adults with hematological malignancies. Stem Cells Dev 22: 1007-1015.

HALADE GV, JIN YF, LINDSEY ML (2013). Matrix metalloproteinase (MMP)-9: a proximal biomarker for cardiac remodeling and a distal biomarker for inflammation. Pharmacol Ther 139: 32-40.

HIRAMATSUH, YAMAMOTOA, KYONOK, HIGASHIYAMAY, FUKUSHIMAC, SHIMA H, SUGIYAMAS, INAKAK and SHIMIZUR (2004). The crystal structure of human dipeptidyl peptidase IV (DPPIV) complex with diprotin A. Biol Chem 385: 561-564.

ITOU M, KAWAGUCHI T, TANIGUCHI E, SATA M (2013). Dipeptidyl peptidase-4: a key player in chronic liver disease. World J Gastroenterol 19: 2298-2306.

KANG MR, YANG G, PLACE RF, CHARISSE K, EPSTEIN-BARASHH, MANOHARAN M and LI LC (2012). Intravesical delivery of small activating RNA formulated into lipid nanoparticles inhibits orthotopic bladder tumor growth. CancerRes 72:5069-5079.

KAWAI T, CHOI U, LIU PC, WHITING-THEOBALD NL, LINTON GF, MALECH HL (2007). Diprotin A infusion into nonobese diabetic/severe combined immunodeficiency mice markedly enhances engraftment of human mobilized CD34+ peripheral blood cells. Stem Cells Dev 16: 361-370.
KERTESZ Z, LINTON EA, REDMAN CW (2000). Adhesion molecules of syncytiotrophoblast microvillous membranes inhibit proliferation of human umbilical vein endothelial cells. Placenta 21: 150-159.

MENKHORST EM, LANE N, WINSHIP AL, LI P, YAP J, MEEHAN K, RAINCZUK A, STEPHENS A, DIMITRIADIS E (2012). Decidual-secreted factors alter invasive trophoblast membrane and secreted proteins implying a role for decidual cell regulation of placentation. PLOS One 7: e31418.

NILSSON E, SALONEN ROS H, CNATTINGIUS S, LICHTENSTEIN P (2004). The importance of genetic and environmental effects for pre-eclampsia and gestational hypertension: a family study. BJOG 111: 200-206.

NISHIKAWA M, ITAKURA A, ITO M, TAKEUCHI M, SATO Y, KAJIYAMA H, MIZU TANI S and KIKKAWA F. (2005). Changes in placental dipeptidyl peptidase IV in preeclampsia with intrauterine growth restriction. Horm Metab Res 37: 408-413.

SATO Y, FUJIWARA H, HIGUCHI T, YOSHIOKA S, TATSUMI K, MAEDA M, FUJII S (2002). Involvement of dipeptidyl peptidase IV in extravillous trophoblast invasion and differentiation. J Clin Endocrinol Metab 87: 4287-4296.

SCHARPÉ S, DE MEESTER I (2001). Peptide truncation by dipeptidyl peptidase IV: a new pathway for drug discovery? Verh K Acad Geneeskd Belg 63: 5-33.

SEHMSDORF US, ZENCLUSSEN AC, ARCK P, HERTWIG K, JOACHIM RA, KLAPP $B$ and HILDEBRANDTMO (2004). Human miscarriage is associated with increased number of CD26 decidual lymphocytes. Scand J Immunol 59: 400-407.

STEEGERS EA, VON DADELSZEN P, DUVEKOT JJ, PIJNENBORG R (2010). Preeclampsia. Lancet 376:631-644.

WESLEY UV, TIWARI S, HOUGHTON AN (2004). Role for dipeptidyl peptidase IV in tumor suppression of human non small cell lung carcinoma cells. Int J Cancer 109: 855-866. 


\section{Further Related Reading, published previously in the Int. J. Dev. Biol.}

Akt1 and insulin-like growth factor 2 (Igf2) regulate placentation and fetal/postnatal development Lindsey N. Kent, Shigeki Ohboshi and Michael J. Soares

Int. J. Dev. Biol. (2012) 56: 255-261

http://dx.doi.org/10.1387/ijdb.113407lk

\section{Local regulation of implantation at the human fetal-maternal interface}

Evdokia Dimitriadis, Guiying Nie, Natalie J. Hannan, Premila Paiva and Lois A. Salamonsen

Int. J. Dev. Biol. (2010) 54: 313-322

http://dx.doi.org/10.1387/ijdb.082772ed

Critical growth factors and signalling pathways controlling human trophoblast invasion Martin Knöfler

Int. J. Dev. Biol. (2010) 54: 269-280

http://dx.doi.org/10.1387/ijdb.082769mk

Trophoblast phagocytic program: roles in different placental systems

Estela Bevilacqua, Mara-Sandra Hoshida, Andrea Amarante-Paffaro, Andrea Albieri-Borges and Sara Zago-Gomes

Int. J. Dev. Biol. (2010) 54: 495-505

http://dx.doi.org/10.1387/ijdb.082761eb

Developmental cell biology of human villous trophoblast: current research problems John D. Aplin

Int. J. Dev. Biol. (2010) 54: 323-329

http://dx.doi.org/10.1387/ijdb.082759ja

A simple in vivo approach to investigate invasive trophoblast cells Juan A. Arroyo, Toshihiro Konno, Darya C. Khalili and Michael J. Soares

Int. J. Dev. Biol. (2005) 49: 977-980

http://dx.doi.org/10.1387/ijdb.051993ja

5 yr ISI Impact Factor $(2011)=2.959$
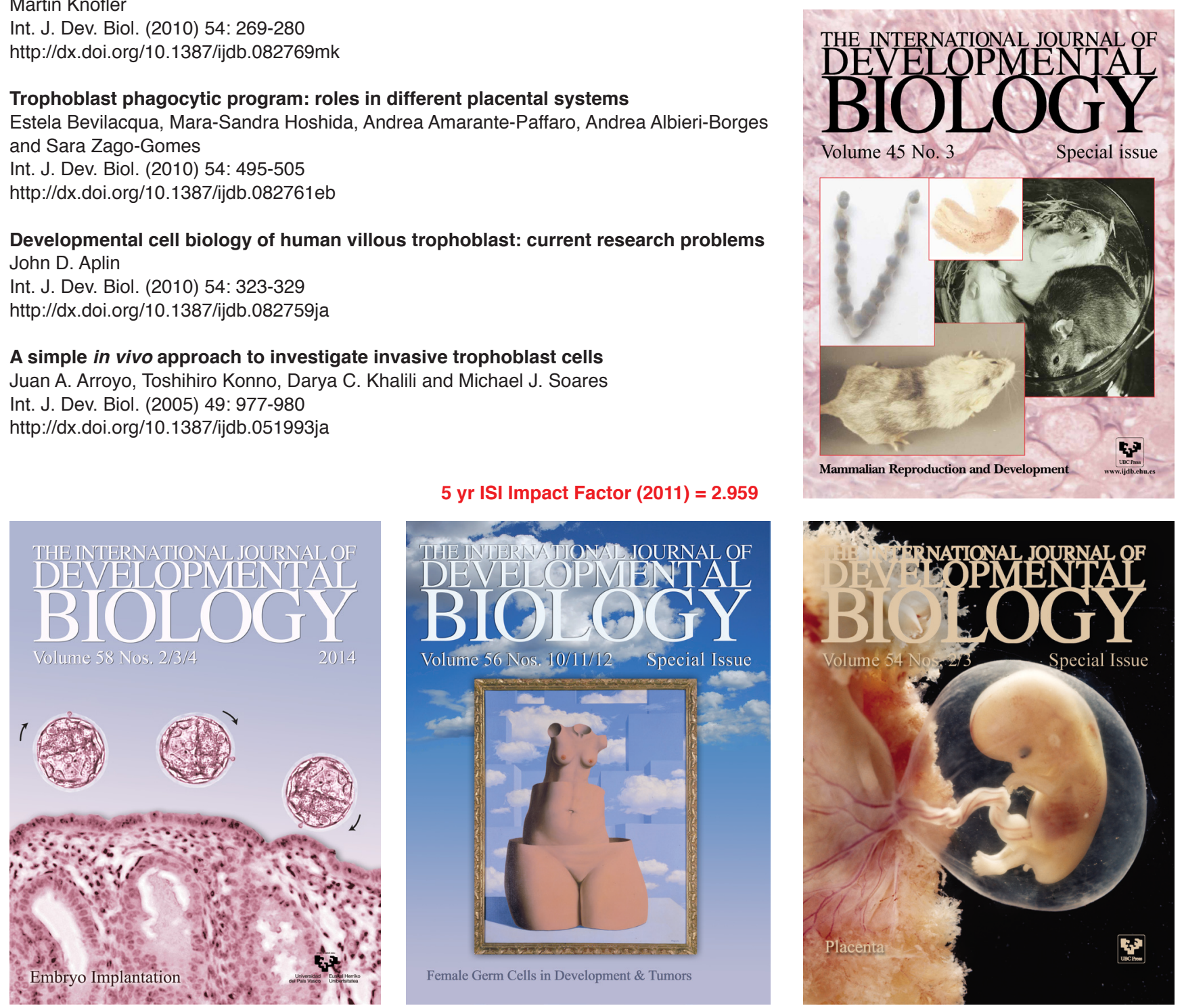